\title{
The thymic stromal cell line MTSC4 induced thymocyte apoptosis in a non- MHC-restricted manner
}

\author{
Xue Ying $\mathrm{HE}^{1}$, Juan $\mathrm{LI}^{1}$, Xiao Ping QIAN ${ }^{1}$, Wen Xian $\mathrm{FU}^{1}$, Yan $\mathrm{LI}^{1}$, Li WU', Wei Feng CHEN ${ }^{1}$ \\ ${ }^{1}$ Department of Immunology, Peking University Health Science Center, 38 Xueyuan Road, Beijing 100083, China. \\ ${ }^{2}$ The Walter and Eliza Hall Institute, PO Royal Melbourne Hospital, Victoria 3050, Australia.
}

\begin{abstract}
Mouse thymic stromal cell line 4 (MTSC4) is one of the stromal cell lines established in our laboratory. While losing the characteristics of epithelial cells, they express some surface markers shared with thymic dendritic cells (TDCs). To further study the biological functions of these cells, we compared the capability of MTSC4 with TDCs in the induction of thymocyte apoptosis, using thymic reaggregation culture system. Apoptosis of thymocytes induced by MTSC4 and TDCs was measured by Annexin V and PI staining and analyzed by flow cytometry. We found that MTSC4 selectively augmented the apoptosis of $\mathrm{CD}^{+} 8^{+}$(DP) thymocytes. This effect was Fas/FasL independent and could not be blocked by antibodies to MHC class I and class II molecules. In addition, MTSC4 enhanced the apoptosis of DP thymocytes from different strains of mice, which implies that MTSC4-induced thymocyte apoptosis is not mediated by the TCR recognition of self peptide/MHC molecules. In contrast to MTSC4, thymocyte apoptosis induced by TDCs was MHCrestricted. Thus, MHC-independent fashion of stromal-DP thymocyte interaction may be one of the ways to induce thymocyte apoptosis in thymus. Our study has also shown that the interaction of MTSC4 stromal cells and thymocytes is required for the induction of thymocyte apoptosis.
\end{abstract}

Keywords: MHC, thymic dendritic cells, thymic stromal cell line, thymocyte apoptosis.

\section{INTRODUCTION}

The communications between thymic microenvironmental stromal cells and thymocytes determine the development and fate of thymocytes. During maturation, the thymocytes interact with the stromal cells, including epithelial cells, fibroblasts and dendritic cells, to undergo $T$ cell receptor (TCR) gene rearrangement and TCR/CD3 expression, thymic selection and functional maturation. Although the detailed molecular mechanism of thymic selection remains unclear, it is generally believed that the positive and negative selection of immature thymocytes depends on the avidity of $\mathrm{T}$ cell receptor (TCR) to peptide/MHC complex and requires secondary signals which are poorly defined. High avidity interaction between TCR and peptide/MHC molecules induces negative selection resulting in cell apoptosis, whereas low avidity interaction between them leads to positive selection for cell survival $[1,2]$. Thymic dendritic cells

"Correspondence: Wei Feng CHEN,

Tel: 0086-10-82802593, Fax: 0086-10-82801436,

E-mail:wfchen@public.bta.net.cn

Abbreviations: BSS, balanced salt solution; DN, double-negative; DP, double-positive; Int, intermediate; MTEC1, mouse thymic epithelial cell line 1; MTSC4, mouse thymic stromal cell line 4; NCS, newborn calf serum; SP, single-positive; TDC, thymic dendritic cell; TRC, thymic reaggregation culture.
(TDCs) as professional antigen presenting cells express high levels of MHC class II molecules and their role in negative selection has been demonstrated in vivo and in vitro $[3,4]$. Apart from TDCs, other types of thymic stromal cells may also be involved in negative selection, such as thymic medullary epithelial cells[5-7].

MTSC4 is a mouse thymic dendritic cell-like cell line established in our laboratory. It was identified by the expression of vimentin and S-100 protein and lacking of characteristic epithelial cell molecules, such as cytokeratin, tonofilaments and desmosomes[8]. Further characterization showed that $70 \%$ of MTSC 4 cells were CD40 positive, express low levels of B7.1, CD8 $\alpha$ and MHC class I and do not express surface MHC class II and B7.2. The proportion of MTSC4 cells expressing DEC-205 varied among the MTSC4 sub-clones (5\%-54\%). However, upon stimulation by a combination of cytokines (IFN- $\gamma$, IL-4, GM-CSF and anti-CD40 mAb), the clone of MTSC4-10 was upregulated on the expression of MHC class I, class II and B7.1. The up-regulation of MHC class II was IFN- $\gamma$ dependent, and omission of any one of the cytokines from the cocktail reduced the up-regulation of B7.1 expression. Our previous studies also showed that application of another cocktail of cytokines, including IL-1, IL-3, SCF and 
Flt3L, did not change the cell phenotype. These results demonstrated that MTSC4 lacked the surface markers typical for thymic epithelial cells and dendritic cells (DCs). However, upon activation, they up-regulated the expression of some surface molecules normally expressed on DCs.

To determine the relationship between MTSC4 and the thymic dendritic cells (TDCs) and to investigate the possible functions of these cells during thymic selection, we compared MTSC4 to freshly isolated TDCs for their capacity to induce thymocyte apoptosis. MTSC4 cells could efficiently augment the apoptosis of $\mathrm{CD}^{+} 8^{+}$thymocytes in a Fas/FasL independent manner. In contrast to TDCs, the induction of thymocyte apoptosis by MTSC4 was not MHC-restricted. These results suggest that different molecular pathways may be involved in the induction of thymocyte apoptosis in MTSC4 and TDCs.

\section{MATERIALS AND METHODS}

\section{Mice}

Six to eight week-old, specific pathogen-free female BALB/c $(\mathrm{H}-$ $\left.2^{\mathrm{d}}\right)$ and $\mathrm{C} 57 \mathrm{BL} / 6\left(\mathrm{H}-2^{\mathrm{b}}\right)$ mice were obtained from the Department of Laboratory Animal Science, Peking University Health Science Center. Six to eight week-old $\mathrm{C} 3 \mathrm{H}\left(\mathrm{H}-2^{\mathrm{k}}\right)$ mice were purchased from Beijing Vital River Laboratory Animal Co., Ltd.

\section{Cell lines}

MTEC1 (mouse thymic medullary epithelial cell line 1), MTSC4 (mouse thymic stromal cell line 4) and its clone, MTSC4-10, were established from the thymus of BALB/c mice in our laboratory $[8$, 9]. 3 T3 fibroblast cell line was purchased from ATCC (VA, USA).

\section{Monoclonal antibodies}

Fluorescence-conjugated monoclonal antibodies used for flow cytometry analysis including: anti-MHC class II I-A(M5/114)-phycoerythrin (PE), anti-CD8 $\alpha$ (53-6.7)-PerCP, anti-CD8 $\alpha$ (53-6.7)Cychrome, anti-CD4(RM4-5)-APC, and anti-CD3(145-2C11)-FITC were purchased from PharMingen (San Diego, CA). Anti-DEC-205 (NLDC-145)-PE was a kind gift given by Dr. Shortman (Melbourne, Australia). Supernatants or ascites of mAbs used for the depletion by immuno-magnetic beads or for cell cultures were generated in our laboratory, including: anti-erythroid cell antigen (Ter-119), antiThy1.2 (30H12), anti-Gr-1(RB68C5), anti-CD3 (KT3-1.1), antiCD4 (RL172), anti-B220 (RA36B2), anti-B7.2 (GL1), anti-CD11c (N418), anti-MHC II I-A (M5/114) and anti-MHC class I (HB-79).

\section{Isolation of thymic dendritic cells (TDCs)}

Thymic DCs were isolated according to Vremec et al [10]. Briefly, thymuses of BABL/c mice were chopped into small fragments with scissors, digested with collagenase type II (1 mg/ml; Worthington) and DNase I (30 U/ml; Worthington) for $25 \mathrm{~min}$, at room temperature with continuous agitation. The cells were washed by EDTA$\mathrm{BSS}$, recovered by centrifugation at $500 \mathrm{~g}$ for $7 \mathrm{~min}$, then resuspended in cold mouse isoosmotic Nycodenz $\left(\mathrm{pH} 7.2\right.$, density $1.077 \mathrm{~g} / \mathrm{cm}^{3}$, containing $5 \mathrm{mM}$ EDTA). The low-density cell fraction was obtained by centrifugation for $10 \mathrm{~min}$ at $1,700 \mathrm{~g}, 4^{\circ} \mathrm{C}$. To deplete nonDC cells, the recovered low-density cells were incubated with a cocktail containing the following mAbs: anti-CD3, anti-CD4, antierythroid cell antigen, anti-Gr-1, anti-B220 and anti-Thy1.2 (1/3 saturation concentration) for $30 \mathrm{~min}$ at $4^{\circ} \mathrm{C}$. Cells were then washed and depleted with anti-Ig-coated magnetic beads (Paesel and Lorei, GMBH \& Co, Frankfurt, Germany) at 10:1 bead-to-cell ratio. The mixture of beads and cells was resuspended in EDTA-BSS-NCS and incubated for $25 \mathrm{~min}$ with continuous slow rotation at $4^{\circ} \mathrm{C}$. To obtain purified TDCs, the cell preparation was stained and sorted for $\mathrm{CD} 11 \mathrm{c}^{+} \mathrm{CD} 8 \alpha^{+}$cells by flow cytometry Vantage (Becton Dic-kinson, Mountain View, CA). The purity of separated TDCs was $95 \%$ after reanalyzed on FACS.

\section{Effects of cytokines on MTEC1, MTSC4 and MTSC4-10}

Murine rIFN- $\gamma$ was purchased from PharMingen. Murine rIL-4, murine rGM-CSF and anti-CD40 mAb (FGK45.5) were obtained from Immunex Corp. (Seattle, WA) and from Dr. A. Rolink (Basel Institute for Immunology). MTEC1, MTSC4 and MTSC4-10 cells $\left(2.5 \times 10^{4}\right.$ cells $\left./ \mathrm{ml}\right)$ were cultured in media supplemented with a cocktail of cytokines containing mrIL-4 (100U/ml), mrGM-CSF (200 ng/ $\mathrm{ml})$, IFN- $\gamma(4 \mathrm{ng} / \mathrm{ml})$ for $48 \mathrm{~h}$, then added anti-CD40 mAb $(1 \mu \mathrm{g} / \mathrm{ml})$ and the cells were cultured for additional $24 \mathrm{~h}$. Cells were then harvested and analyzed by FACS Calibur (Becton Dickinson, Mountain View, CA).

\section{Preparation of thymic subsets}

Fresh adult thymuses were homogenized to a single-cell suspension in cold RPMI- 1640 medium containing $2 \%$ newborn calf serum (NCS), using steel mesh. The cells were stained with anti-CD8 $\alpha-$ Cychrome, anti-CD4-APC, and sorted for $\mathrm{CD}^{+} 8^{+}(\mathrm{DP}), \mathrm{CD}^{+} \mathrm{CD} 8^{-}$ (CD4SP) and CD4-CD8 ${ }^{+}$(CD8SP) cells by flow cytometry on FACS Vantage. The purity of separated thymocyte subsets was $97-98 \%$ after reanalyzed on FACS.

\section{Thymic reaggregation cultures (TRC)}

The TRC was based on the method established by Anderson et al. [4]. Freshly prepared thymocytes and appropriate stromal cells were mixed together at necessary ratios and pelleted by centrifugation. Following removal of the supernatant, the cell pellet was carefully transferred to the surface of a $0.8 \mu \mathrm{m}$ Nucleopore filter supported by gelatin foam sponges.

\section{Flow cytometric analysis}

MTSC4 and MTSC4-10 cells were dispersed in 0.1\% EDTAPBS ( $\mathrm{pH} \mathrm{8.0)}$ at $37^{\circ} \mathrm{C}$ for $15 \mathrm{~min}$. After blocking with mouse and rat sera, the single cell suspensions were stained with a panel of conjugated $\mathrm{mAbs}$ (see Monoclonal antibodies) for $30 \mathrm{~min}$ at $4^{\circ} \mathrm{C}$. Stained cells were analyzed on FACS Calibur. Data acquisition and analysis were performed using Cell Quest software.

Annexin-V-FITC kit (BD-PharMingen) was used to evaluate thymocyte apoptosis. Thymocytes were harvested after reaggregation cultures and stained with Annexin V-FITC and PI, simultaneously with CD8-PerCP and CD4-APC, then analyzed by FACS to determine the proportion of apoptotic cells at different stages and the viable cells as well as the four major thymocyte subsets in the viable cells. 
A

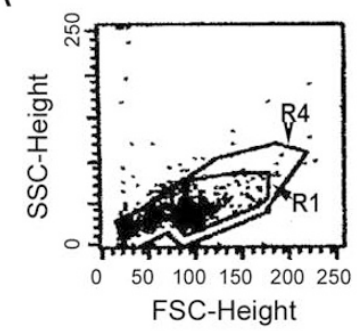

B

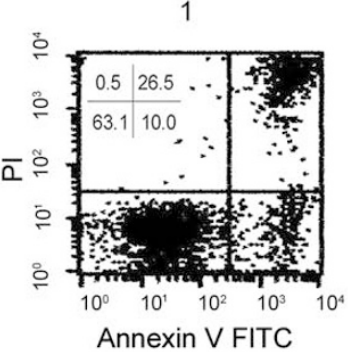

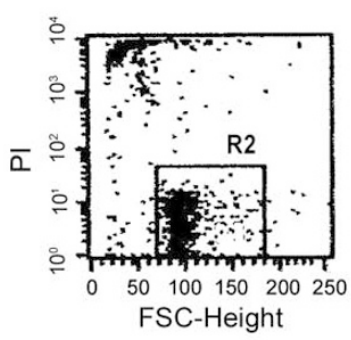

2

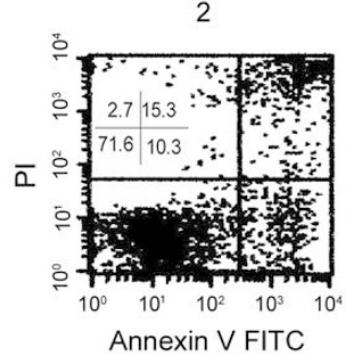

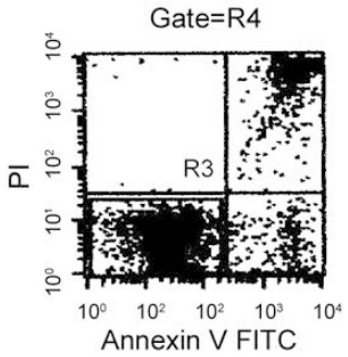

3

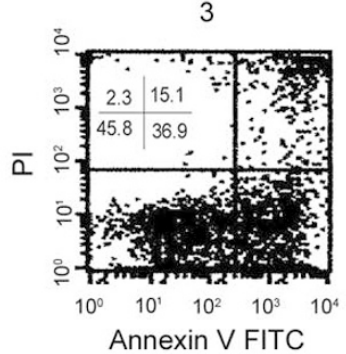

Fig 1. MTSC4 cells induced thymocyte apoptosis. Thymocytes were cultured in TRC system in the presence or absence of MTSC4 cells for $12 \mathrm{~h}$. The cells were stained with Annexin V and PI, then, analyzed on FACS to determine the apoptotic cells at distinct stages and the proportion of viable cells. MTEC1 cells were applied as control and cocultured with thymocytes under the same condition as that of MTSC4 cells. (A) Gates and regions used on FACS profiles for analyzing the viability of cultured thymocytes. R1: forward scatter gating for viable cells; R2: gating for PI- cells; R3: gating for Annexin $\mathrm{V}^{-}$ $\mathrm{PI}^{-}$cells; R4: total cells recovered from each culture. (B) Effects of MTSC4 and MTEC1 on thymocyte apoptosis. $5 \times 10^{5}$ thymocytes were co-cultured with MTSC4 or MTEC1 at the ratio of 1:2 in number. Thymocytes were harvested from these cultures after $12 \mathrm{~h}$, and the viable cells were counted. The number of viable cells to total cells recovered from these cultures was: thymocytes alone: $2.7 / 3.6 \times 10^{5}$; MTEC1+thymocytes: $2.4 / 3.0 \times 10^{5}$; MTSC4+thymocytes: $2.2 / 3.2 \times 10^{5}$. The harvested thymocytes were then stained with Annexin V-FITC and PI to reveal the apoptotic thymocytes. 1. Thymocytes alone; 2. MTEC1 + thymocytes; 3 . MTSC4 + thymocytes. A representative experiment of three is shown.

\section{Antibody blocking assays}

To determine the functions of MHC molecules, Fas/FasL, and CD40 in thymocyte apoptosis induced by MTSC4, mAbs to MHC class I (ascites, 1:400) and MHC class II (ascites, 1:100), anti-CD40 (HM40-3, PharMingen) $(10 \mu \mathrm{g} / \mathrm{ml})$, and anti-FasL $(10 \mu \mathrm{g} / \mathrm{ml})$ (MFL3, PharMingen) were added into the TRCs respectively. Thymocyte apoptosis was assayed by Annexin V and PI staining, and analyzed by FACS. The functions of MHC molecules in thymocyte apoptosis induced by TDCs were also investigated.

\section{RESULTS}

\section{Thymocyte apoptosis induced by MTSC4 and TDCs}

Mouse thymocytes underwent spontaneous apoptosis in vitro. In TRC system, thymocyte apoptosis could be revealed after being cocultured with MTSC4 cells for $12 \mathrm{~h}$. To determine the capacity of MTSC4 in the induction of apoptosis, $1 \times 10^{6}$ MTSC 4 cells were co-cultured with $5 \times 10^{5}$ thymocytes isolated from $\mathrm{BALB} / \mathrm{c}$ mice for $12 \mathrm{~h}$ at $37^{\circ} \mathrm{C}$. MTEC1, a thymic epithelial cell line which was shown to positively support thymocyte development[11], was used in the co-cultures with thymocytes as a control. Cultures with thymocytes alone were used as controls for spontaneous apoptosis. The effect of MTSC4 on thymocyte apoptosis and the subsets of the viable thymocytes were examined by staining with Annexin V-FITC, PI, CD8PerCP, CD4-APC and analyzed on flow cytometry. The electronic gatings used for analyzing the viability of the cultured cells were shown in Fig 1A. The viable cells were defined as Annexin $\mathrm{V}^{-} \mathrm{PI}^{-}$; the early phase of apoptotic cells defined as Annexin $\mathrm{V}^{+} \mathrm{PI}^{-}$; and the intermediate phase of apoptotic cells as Annexin $\mathrm{V}^{+} \mathrm{PI}^{+}$cells. As shown in Fig $1 \mathrm{~B}$, the apoptotic cells were $36.9 \%, 28.3 \%$ and $54.2 \%$ in the thymocytes cultured alone, cultured with MTEC1 or MTSC4, respectively. The apoptotic cells increased by $47 \%$ in the cocultures with MTSC4, whereas decreased by $23 \%$ in the cocultures with MTEC1. Thus, MTSC4 promoted thymocyte apoptosis; in contrast, MTEC1 rescued thymocyte apoptosis, the later was consistent with our previous observation[11, 12].

MTSC4-10 is a subclone of MTSC4[9]. Upon stimulation by GM-CSF, IL- 4 , IFN- $\gamma$ and anti-CD $40 \mathrm{mAb}$, MTSC4-10 up-regulated the expression of surface MHC 
Tab 1. Effects of MTSC4, MTSC4-10 and cytokine-stimulated MTSC4-10 cells on the induction of thymocyte apoptosis

\begin{tabular}{|c|c|c|c|c|c|c|c|c|c|}
\hline \multirow[t]{2}{*}{ Groups } & \multicolumn{2}{|c|}{ Cell number $\left(\times 10^{5}\right)$} & \multicolumn{3}{|c|}{ Apoptotic cells (\%) } & \multicolumn{4}{|c|}{ Viable cell subsets $(\%)$} \\
\hline & (viable/total) & ${ }^{2} \mathrm{Ann}^{-} \mathrm{PI} \mathrm{I}^{-}$ & $\mathrm{Ann}^{+} \mathrm{PI}^{-}$ & $\mathrm{Ann}^{+} \mathrm{PI}^{+}$ & $\mathrm{Ann}^{-} \mathrm{PI}^{+}$ & $\mathrm{CD}^{+} \mathrm{SP}$ & $\mathrm{CD}^{+} \mathrm{SP}$ & $\mathrm{CD} 4^{+} 8^{+}$ & $\mathrm{CD} 48^{-}$ \\
\hline${ }^{1}$ Thy (0h) & ${ }^{4} \mathrm{ND}$ & ND & ND & ND & ND & 11.7 & 4.0 & 79.7 & 4.6 \\
\hline Thy & $2.5 / 3.3$ & 60.0 & 14.0 & 24.2 & 1.7 & 12.1 & 4.0 & 82.1 & 1.8 \\
\hline Thy+MTSC4 & $2.0 / 3.1$ & 44.6 & 23.2 & 23.9 & 8.3 & 19.6 & 7.6 & 65.9 & 6.9 \\
\hline Thy+MTSC4-10 & $2.3 / 3.4$ & 47.8 & 18.3 & 28.6 & 5.3 & 18.9 & 7.6 & 65.5 & 8.0 \\
\hline${ }^{3}$ Thy+MTSC4-10s & $2.1 / 3.2$ & 45.9 & 14.5 & 35.9 & 3.7 & 17.9 & 6.0 & 67.7 & 8.5 \\
\hline
\end{tabular}

$5 \times 10^{5}$ thymocytes were cocultured with MTSC4 cell line and clones at ratio of 1:2. Cells were harvested after $12 \mathrm{~h}$ for analysis. ${ }^{1}$ Thy: thymocytes; ${ }^{2}$ Ann: Annexin V; ${ }^{3}$ MTSC4-10s: MTSC4-10 stimulated by cytokine cocktail (GM-CSF, IL-4, IFN- $\gamma$ ) and anti-CD40 $\mathrm{mAb} ;{ }^{4} \mathrm{ND}$ : not done. A representative experiment of three is shown.

DP

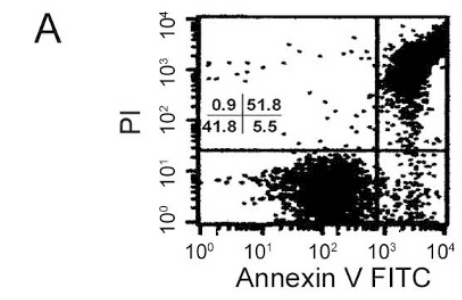

B
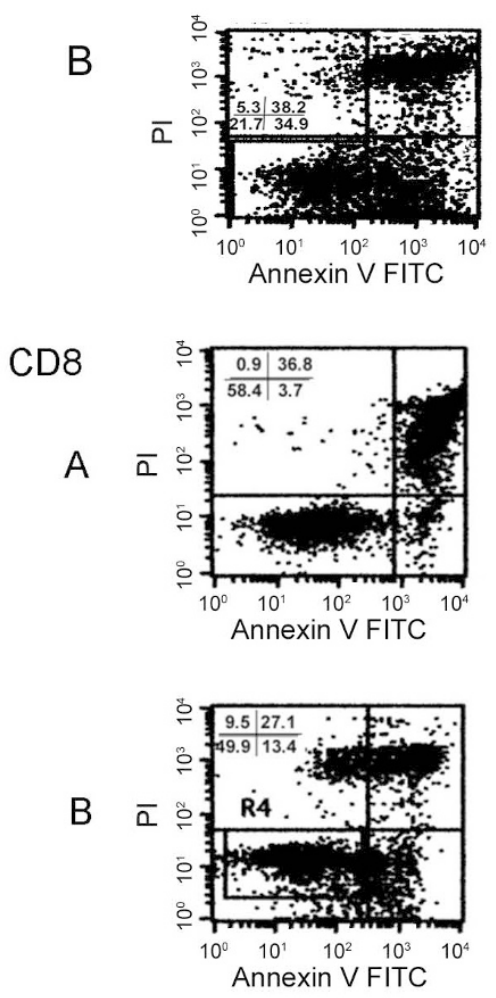
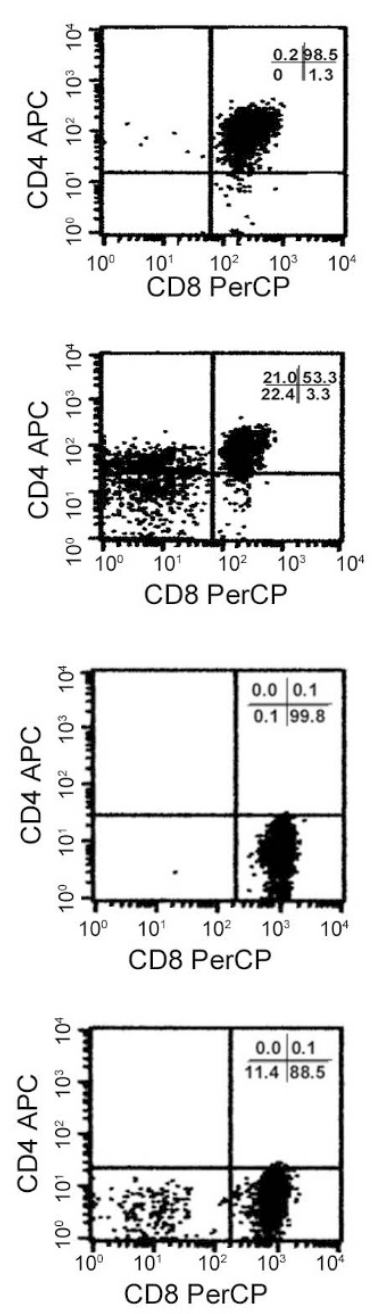

class I, class II and B7.1 molecules. However, the higher levels of expression of these molecules on MTSC4-10 did not seem to enhance their ability to induce thymocyte apoptosis in comparison with MTSC4. As shown in Tab 1, MTSC4, MTSC4-10 and the cytokine-stimulated MTSC410 cells induced thymocyte apoptosis with similar

CD4
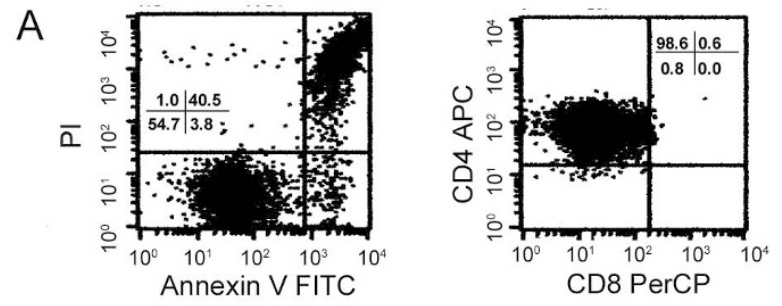

B
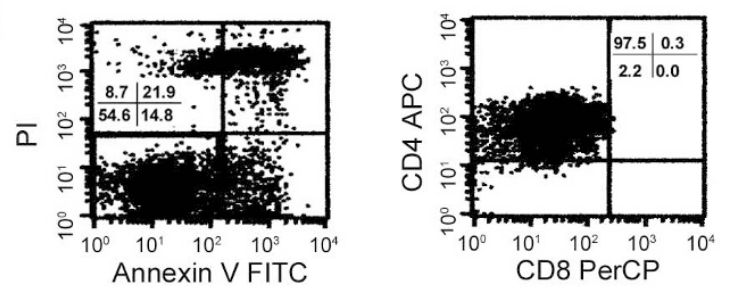

Fig 2. The susceptibility of apoptosis of thymocyte subsets induced by MTSC4 cells. Thymocytes of DP, CD4SP and CD8SP subsets were isolated and cocultured in TRC system with MTSC4 cells for $12 \mathrm{~h}$, then the cells were stained with Annexin-V-FITC and PI, followed by staining with anti-CD8 $\alpha$ (53-6.7)-PerCP and anti-CD4 (RM4-5)APC for 4-color analysis on FACS to determine the apoptotic cells at different stages and the phenotypes of the viable cells, (A) DP, CD4SP, CD8SP cells cultured alone for $12 \mathrm{~h}$; (B) DP, CD4SP, CD8SP thymocytes cocultured with MTSC4 for $12 \mathrm{~h}$. The right profiles of all groups represent the phenotypes of viable cells after culture alone or cocultured with MTSC4. 
A

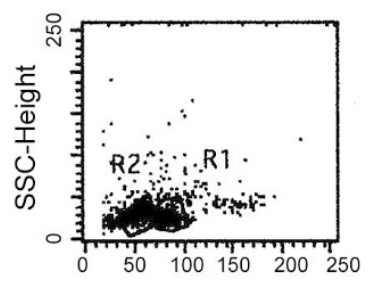

B

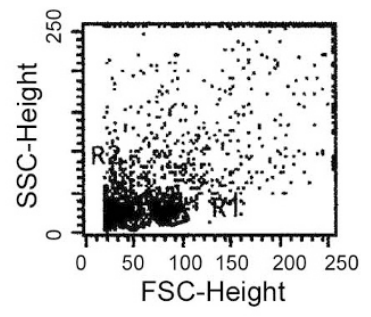

Gate=R2
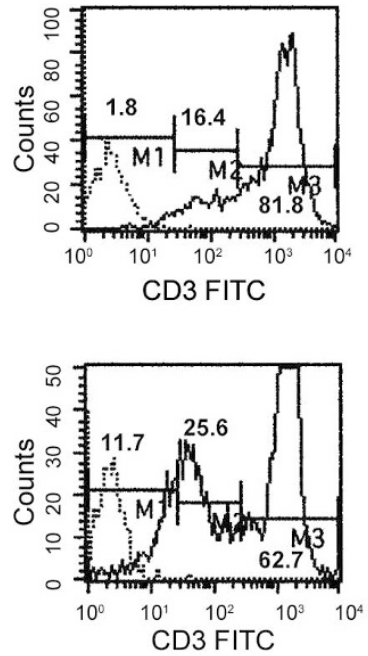

Gate=R1
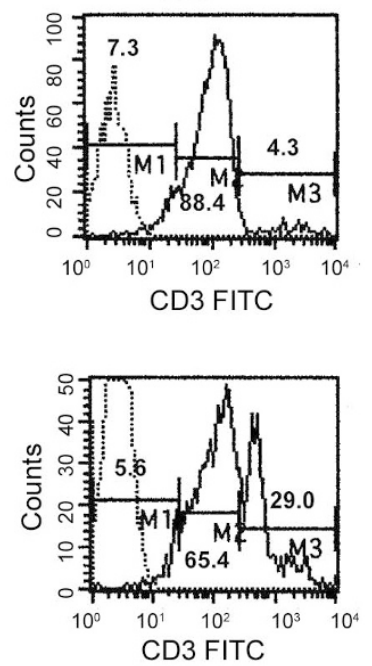

Fig 3. Expression of CD3 on viable and apoptotic DP thymocytes after $12 \mathrm{~h}$ in vitro TRC system cultures with the absence or presence of MTSC4. The viable cells were gated by R1, and the apoptotic cells gated by R2. (A) DP cells cultured alone; (B) DP cells cocultured with MTSC4. Dotted line, isotype control; solid line, anti-CD3 (145-2C11)-FITC mAb stained cells. In the DP cells cultured alone, the $\mathrm{CD} 3$ negative, intermediate and high positive cells were $1.8 \%, 16.4 \%$ and $81.8 \%$, respectively. In the DP cells cocultured with MTSC4, the CD3 negative, intermediate and high positive cells were $11.7 \%, 25.6 \%$ and $62.7 \%$, respectively.

Tab 2. MTSC4 induced apoptosis of thymocytes isolated from C57BL/6 and $\mathrm{C} 3 \mathrm{H}$ mice

\begin{tabular}{|c|c|c|c|c|c|c|c|c|c|}
\hline \multirow[t]{2}{*}{ Groups } & \multirow{2}{*}{$\begin{array}{l}\text { Cell number }\left(\times 10^{5}\right) \\
(\text { viable/total })\end{array}$} & \multicolumn{4}{|c|}{ Apoptotic cells (\%) } & \multicolumn{4}{|c|}{ Viable cell subsets $(\%)$} \\
\hline & & Ann $^{-} \mathrm{PI}^{-}$ & ${ }^{2} \mathrm{Ann}^{+} \mathrm{PI}^{-}$ & $\mathrm{Ann}^{+} \mathrm{PI}^{+}$ & $\mathrm{Ann}^{-} \mathrm{PI}^{+}$ & $\mathrm{CD} 4^{+} \mathrm{SP}$ & $\mathrm{CD} 8^{+} \mathrm{SP}$ & $\mathrm{CD} 4^{+} 8^{+}$ & $\mathrm{CD} 48^{-}$ \\
\hline \multicolumn{10}{|c|}{ Thymocytes from C57BL/6 mice } \\
\hline${ }^{1}$ Thy $(0 \mathrm{~h})$ & ${ }^{3} \mathrm{ND}$ & ND & ND & ND & ND & 15.0 & 3.5 & 79.0 & 2.5 \\
\hline Thy alone & $2.2 / 2.8$ & 72.5 & 12.6 & 11.2 & 3.7 & 12.0 & 3.9 & 82.1 & 2.0 \\
\hline Thy+MTSC4 & $2.6 / 3.3$ & 57.3 & 20.7 & 12.0 & 10.0 & 16.1 & 5.0 & 69.6 & 9.4 \\
\hline \multicolumn{10}{|c|}{ Thymocytes from $\mathrm{C} 3 \mathrm{H}$ mice } \\
\hline Thy $(0$ h) & ND & ND & ND & ND & ND & 13.0 & 3.9 & 80.1 & 3.0 \\
\hline Thy alone & $2.3 / 3.0$ & 57.6 & 12.5 & 27.8 & 2.1 & 12.1 & 4.0 & 82.1 & 1.8 \\
\hline Thy+MTSC4 & $2.4 / 3.4$ & 47.3 & 20.4 & 29.2 & 3.2 & 12.7 & 5.0 & 69.0 & 14.9 \\
\hline
\end{tabular}

$5 \times 10^{5}$ thymocytes were cocultured with different cell lines at ratio of 1:2. Cells were harvested after $12 \mathrm{~h} .{ }^{1}$ Thy: thymocytes; ${ }^{2}$ Ann: Annexin V; ${ }^{3} \mathrm{ND}$ : not done. A representative experiment of three is shown.

of MTSC4 cells and freshly isolated TDCs to induce thymocyte apoptosis using TRC assays. In order to form TRC efficiently, 3T3, a fibroblast cell line, was used as supporting cells. TRCs were established with thymocytes and $3 \mathrm{~T} 3$ cells at a ratio of 1:2. When TDCs were added at a $10 \%$ of the total cell number in each TRC, $51 \%$ thymocytes became apoptotic after $12 \mathrm{~h}$ culture. This was $80 \%$ higher than that found in control cultures in the absence of TDCs. Using the same system, 10\% MTSC4 cells had no detectable effect on thymocyte apoptosis; whereas when $50 \%$ MTSC4 cells were added, the percentage of apoptotic thymocytes was $53 \%$ higher than that of control cultures. These results demonstrated that TDCs were at least 5-fold more potent than MTSC4 cells in the induction of thymocyte apoptosis.

\section{MTSC4 mainly induced DP thymocytes to apoptosis}

The FACS sorted three thymocyte subsets of DP, CD4SP, CD8SP cells were cocultured with MTSC4 in TRC and the MTSC4 induced thymocyte apoptosis was 


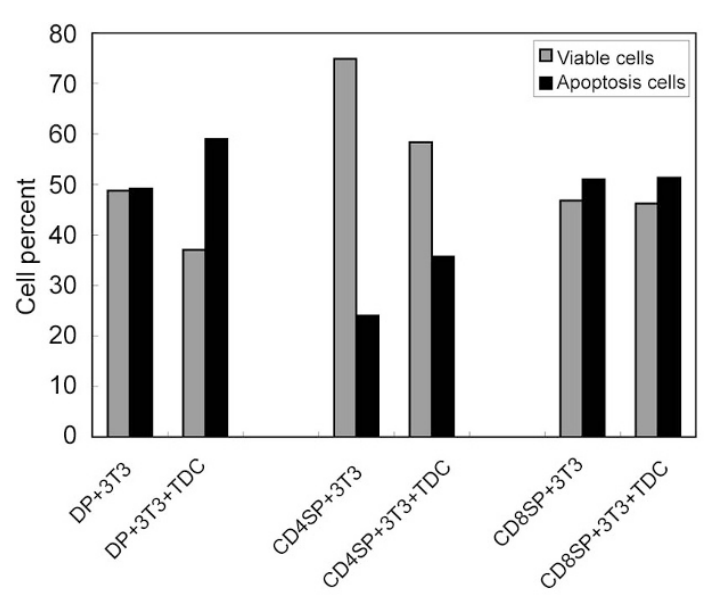

Fig 4. Thymic dendritic cell-induced apoptosis of thymocyte subsets. Purified thymocytes of DP, CD4SP, and CD8SP subsets were cocultured with freshly isolated and purified TDCs in TRC system with the addition of $3 \mathrm{~T} 3$ cells to form reaggregation, then, cultured for $12 \mathrm{~h}$ and the cell apoptosis was analyzed on FACS.

investigated. The results demonstrated that MTSC4 mainly induced DP thymocytes apoptosis. In the cocultures with MTSC4, the percentage of viable DP cells (Annexin V-PI double negative) decreased from $41.8 \%$ to $21.7 \%$, comparing with the DP cells cultured alone. Surprisingly, $45.2 \%$ of the viable cells ( in DP cells cultured alone, the viable cells was $98.5 \%$, whereas in the DP cells cocultured with MTSC4, the viable DP cells were 53.3\%) down-regulated the CD4 and CD8 expression resulted in the enrichment of DN cells $(22.4 \%)$ and the generation of CD4SP thymocytes (21.0\%) (Fig 2). CD8SP cells were mildly but substantially induced to apoptosis by MTSC4, and $11.4 \%$ of the cells down-regulated CD8 to DN phenotype. Meanwhile, the apoptosis of CD4SP cells was not augmented by MTSC4. Importantly, we found that $\mathrm{CD} 3^{- \text {-int }} \mathrm{DP}$ cells induced to apoptosis by MTSC4 were $19.1 \%$ higher compared with that of DP cells cultured alone (calculated from the data shown in Fig 3).

In striking contrast, TDCs induced both DP and CD4SP thymocytes to apoptosis, but appeared to have no effect on CD8SP thymocyte apoptosis (Fig 4).

\section{Induction of thymocyte apoptosis by MTSC4 was not $\mathrm{MHC}$ restricted}

A key factor in determining whether thymocytes undergo positive or negative selection is the avidity of its TCR-mediated specific interaction with peptide/MHC complexes on thymic stromal cells $[1,14,15]$. To test whether MTSC4 induced thymocyte apoptosis was mediated via specific interactions of TCR and peptide/MHC complexes, thymocytes from different strains of mice including C57BL/6
$\left(\mathrm{H}-2^{b}\right)$ and $\mathrm{C} 3 \mathrm{H}\left(\mathrm{H}-2^{\mathrm{k}}\right)$ were reaggregated with MTSC4 cells of BALB/c origin $\left(\mathrm{H}-2^{\mathrm{d}}\right)$, using the same TRC culture conditions. Surprisingly, MTSC4 cells could also induce apoptosis of thymocytes from $\mathrm{C} 57 \mathrm{BL} / 6$ and $\mathrm{C} 3 \mathrm{H}$ mice with similar efficiency as that from BALB/c mice (Tab 2 ). The effects of MTSC4 cells on the apoptosis of thymocytes from mice of different strains, were also mainly on $\mathrm{CD}^{+} 8^{+}$thymocyte subset. In contrast, TDCs from $\mathrm{BALB} / \mathrm{c}$ mice could not induce the apoptosis of thymocytes from C57BL/6 mice. The percentage of apoptotic C57BL/6 thymocytes induced by Balb/c TDC in the co-cultures was $43.4 \%$, compared to $47 \%$ when thymocytes were cultured alone (data not shown). These results indicated that MTSC4 induced thymocyte apoptosis in a non-MHC-restricted manner. In order to confirm this finding, we further tested whether anti-MHC class I and class II antibodies could block the thymocyte apoptosis induced by MTSC4 cells. As shown in Fig 5A, mAbs to MHC molecule (both class-I and II) failed to block the thymocyte apoptosis induced by MTSC4 cells. In contrast, the thymocyte apoptosis induced by TDCs was efficiently blocked by the mAbs against MHC molecules (Fig 5B).

To exclude the possibility that MTSC4 cells might produce certain factors which could be cytotoxic to thymocytes, the culture supernatants of MTSC4 alone and MTSC4 cocultured with thymocytes were collected and added into the thymocyte cultures. No significant thymocyte apoptosis was induced by the supernatants (data not shown), indicating that the induction of thymocyte apoptosis by MTSC4 was not caused by soluble factors, but required cell-cell contact.

\section{Thymocyte apoptosis induced by MTSC4 was Fas/ FasL independent}

MTSC4 cells were shown previously to express FasL [16]. To test whether thymocyte apoptosis induced by MTSC4 was Fas/FasL dependent, MTSC4 cells were incubated with anti-FasL Ab (MFL3) for $1 \mathrm{~h}$ at $4^{\circ} \mathrm{C}$. The anti-FasL $\mathrm{Ab}$ was also added into TRC cultures. Cells were harvested after $12 \mathrm{~h}$ and thymocyte apoptosis was assessed. As shown in Tab 3, anti-FasL antibody had virtually no effect on thymocyte apoptosis induced by MTSC4. Similarly, thymocyte apoptosis induced by TDCs was not affected by anti-FasL antibody (data not shown).

\section{DISCUSSION}

MTSC4, a mouse thymic stroma derived cell line, does not own the characteristic of fibroblasts or epithelial cells, but share some cell surface markers with TDCs, while lack of $\mathrm{CD} 11 \mathrm{c}[8,17]$. Our previous studies showed that upon stimulation with a combination of cytokines, MTSC4 

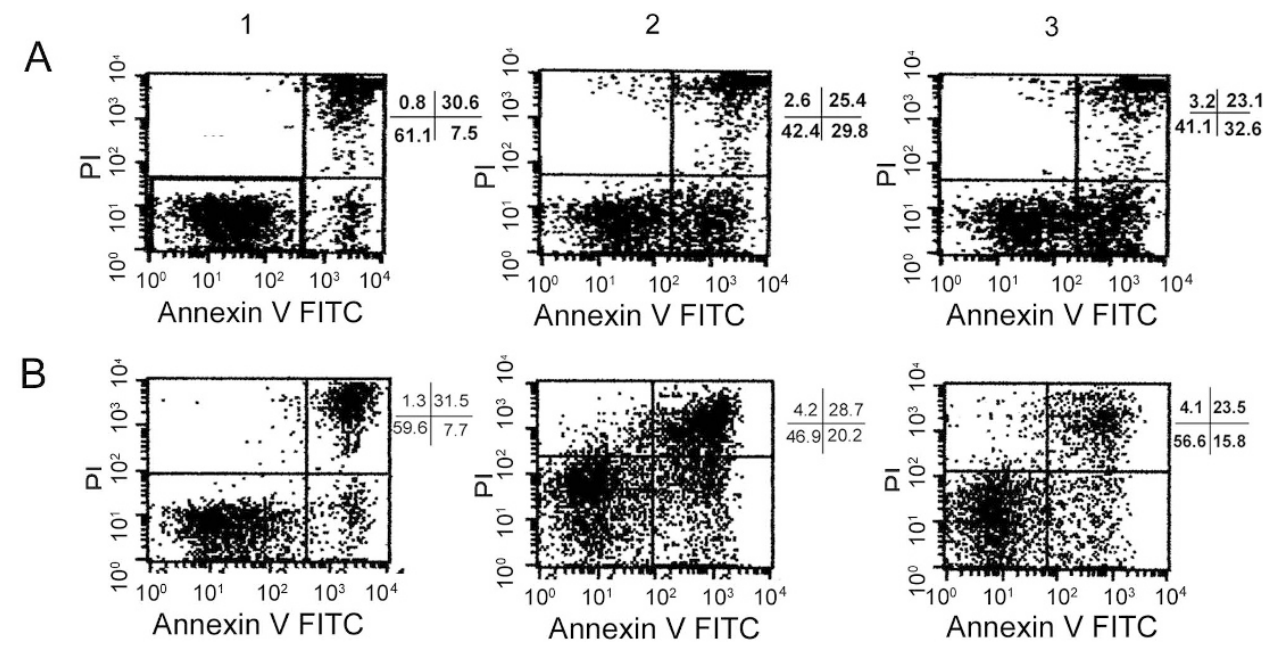

Fig 5. Effect of mAbs to MHC molecules on the blocking of thymocyte apoptosis induced by MTSC4 or TDCs. MTSC 4 cells were incubated at $4^{\circ} \mathrm{C}$ with mAbs of anti-MHC class I (1:400) and II (1:100) for $1 \mathrm{~h}$, then the TRC system was set up in the presence of the mAbs for $12 \mathrm{~h}$ and the proportion of cell apoptosis was analyzed to determine the Ab blocking effect. (A) Thymocytes cocultured with MTSC4. 1. thymocytes alone; 2. thymocytes and MTSC4 in the ratio of 1:2; 3. mAbs to MHC molecules were added in the medium of TRC set up as 2. ( B) Thymocytes cocultured with TDCs. 1. Thymocytes alone; 2. Thymocytes and 3T3 cells in the ratio of 1:2 and 10\% TDCs reaggregated; 3. MAbs to MHC molecules were included in the TRC set up as 2. A representative experiment of three is shown in both $\mathrm{A}$ and $\mathrm{B}$.

Tab 3. No blocking effect of anti-FasL antibody on thymocyte apoptosis inducted by MTSC4

\begin{tabular}{|c|c|c|c|c|c|c|c|c|}
\hline \multirow[t]{2}{*}{ Groups } & \multicolumn{4}{|c|}{ Apoptotic cells (\%) } & \multicolumn{4}{|c|}{ Viable cell subsets $(\%)$} \\
\hline & ${ }^{2} \mathrm{Ann}^{-} \mathrm{PI}^{-}$ & $\mathrm{Ann}^{+} \mathrm{PI}^{-}$ & $\mathrm{Ann}^{+} \mathrm{PI}^{+}$ & $\mathrm{Ann}^{-} \mathrm{PI}^{+}$ & $\mathrm{CD}^{+} \mathrm{SP}$ & $\mathrm{CD}^{+} \mathrm{SP}$ & $\mathrm{CD} 4^{+} 8^{+}$ & $\mathrm{CD} 48^{-}$ \\
\hline${ }^{1}$ Thy & 63.5 & 9.4 & 26.4 & 0.7 & 10.4 & 3.2 & 85.2 & 1.2 \\
\hline Thy+anti-FasL $(10 \mu \mathrm{g} / \mathrm{ml})^{*}$ & 61.4 & 11.3 & 26.8 & 0.5 & 10.0 & 3.8 & 84.9 & 1.2 \\
\hline Thy+MTSC4 & 46.0 & 36.8 & 15.2 & 2.1 & 12.7 & 3.9 & 71.9 & 11.5 \\
\hline Thy+MTSC4+ anti-FasL $(10 \mu \mathrm{g} / \mathrm{ml})$ & 44.5 & 36.6 & 16.3 & 2.6 & 13.7 & 4.6 & 71.2 & 10.5 \\
\hline
\end{tabular}

There were no effects on thymocyte apoptosis by different concentrations $(0.5 \mu \mathrm{g} / \mathrm{ml}, 2.0 \mu \mathrm{g} / \mathrm{ml}$ and $10 \mu \mathrm{g} / \mathrm{ml})$ of anti-FasL antibody. ${ }^{1}$ Thy: thymocytes; ${ }^{2} \mathrm{Ann}$ : Annexin V; *: it represents the final concentration of anti -FasL. A representative experiment of three is shown.

cells up-regulated the expression of MHC class-II and B7.1 (CD80). Although the expression of some surface molecules is common to DCs, MTSC4 cells are not as efficient antigen presenting cells as DCs[18]. In this study, we further investigated the capacity of MTSC4 cells to induce thymocyte apoptosis in comparison with TDCs.

Using TRC assay, we examined the abilities of MTSC4 and its subclone MTSC4-10 in the induction of thymocyte apoptosis. TDCs and MTEC1, a thymic epithelial cell line, were used as controls. Annexin V vs PI staining was used for identifying apoptotic cells at different phases. In order to determine which thymocyte subsets were affected, the harvested cells were also stained with anti-CD4 and antiCD8 mAbs. The percentage of viable thymocyte population was determined by gating for Annexin $\mathrm{V}^{-} \mathrm{PI}^{-}$cells. Our results showed that MTSC 4 cells substantially induced thymocyte apoptosis in TRCs, and the apoptotic cells were mainly in $\mathrm{CD}^{+} 8^{+}$thymocyte subset and in a tiny proportion of CD8 SP thymocytes (Fig 1 and Fig 2). Surprisingly, apart from inducing DP thymocyte apoptosis, MTSC4 cells were also found to induce down-regulation of CD4 and CD8 expression that resulted in the enrichment of viable DN cells and generation of CD4SP thymocytes. The downregulation of CD8 molecules also occurred in MTSC4 induced apoptosis of CD8SP thymocytes (Fig 2). These findings imply that MTSC4 cells may have distinct functions on thymocytes in vitro: to promote thymocyte apoptosis and induce DP to differentiate into CD4SP thymocytes. MTSC4 appeared to have no effect on CD4SP cell apoptosis, but had minor effect on the promotion of CD8SP 
cell apoptosis. These results demonstrate that MTSC4 cells induced thymocyte apoptosis mainly on DP stage.

TDCs play a key role in thymic negative selection. In our TRC assay system, TDCs displayed strong ability to induce thymocyte apoptosis mediated by TCR-self peptide/MHC interaction and blocked by the mAbs to MHC class I and class II molecules as we had expected. In comparison with TDCs, MTSC4 cells are at least 5-fold less efficient in the induction of thymocytes apoptosis. More importantly, there was a qualitative difference in the ability to induce thymocyte apoptosis by MTSC4 and TDCs. The differences are displayed in two aspects: 1) MTSC4 cell induced thymocyte apoptosis is not MHC restricted. The apoptosis extent induced by MTSC4 in thymocytes from different mouse strainsat were similar. In contrast, TDCs induced thymocyte apoptosis is MHC restricted. The non-MHC restricted fashion might be explained by the low expression of MHC class I and class II on MTSC4. 2) MTSC4 mainly induced apoptosis on DP thymocytes; Iy contrast, TDCs induced apoptosis in both DP and CD4SP thymocytes. Apparently, MTSC4 cells induced thymocyte apoptosis was not mediated by the signal derived from TCR-self peptide-MHC interaction, which is required for TDCs induced thymocyte apoptosis. Our assays also excluded the possibility that MTSC4 induced thymocyte apoptosis might be mediated by soluble factors, such as TNF- $\alpha$ and steroid hormones which might be produced by MTSC4 cells. Therefore, cell-cell interaction is required for MTSC4 cells induced DP thymocyte apoptosis.

The MTSC4 cell-induced apoptotic process is not activated by the signal generated from TCR recognition of self peptide/MHC molecules, but is activated by the unknown signals generated from stromal-DP thymocyte interaction, such mechanism may be involved in cell death caused by 'neglect'. The 'neglect' has been proposed as the lack of signals for positive selection and resulted in the majority of cell death in thymus[19]. Whether the cell death by 'neglect' is a simple consequence of no TCR signal remains controversial. Guo et al found that the lifespan of $\mathrm{CD}^{+} 8^{+}$thymocyte in $\mathrm{Bcl}-\mathrm{x}(\mathrm{L})$-transgenic mice was extended, and they concluded that the programmed cell death of $\mathrm{CD}^{+} 8^{+}$thymocytes was not simply a consequence of failed positive selection (20). As around 50\% of DP thymocytes (34\% in our assay) do not express TCR, their death is caused by 'neglect'. The critical issue of the argument is if the 'neglect'caused cell death is a passive consequence, or an active process initiated by the signals derived of DP cell-stromal cell interactions. Our experiments have provided evidence that at least part of the TCR $\alpha \beta^{-}\left(\mathrm{CD}^{-/ \text {int }}\right)$ DP thymocyte apoptosis was triggered by cell-cell interaction. Thereby, thymic stromal cells are likely to be taken part in the activation of cell death by 'neglect'.

The results presented here are based on in vitro TRC assay system. What if the thymic stromal cell-induced apoptosis of non-MHC restriction pattern really happened in vivo? With respect to our primary data that a scFv Ab screened out from human phage-displayed Ab library by MTSC4 cell line could react with a subset of stromal cells within mouse thymus. The MTSC4-like cells might be present in the thymus, and the induction of thymocyte apoptosis by thymic stromal cells in a non-MHC restriction manner might occur in vivo. Further experiments are needed to test its authenticity.

A further question is what sort of ligand-receptor interactions trigger cell death by 'neglect'. There are numerous co-receptors such as CD4, CD8, CD80, CD86, CD40, adhesion molecules such as LFA-1, ICAM-1[21, 22], and other molecules which were reported to provide co-stimulatory signals to augment the TCR-peptide-MHC signal for the activation of programmed cell death[2, 23, 24]. We show here that Fas/FasL is not involved in the apoptosis induced by MTCS4, and anti-CD40 mAb could not interfere with the MTSC4 induced thymocyte apoptosis (data not shown). Further investigation on molecules that may be involved in MTSC4 induced thymocyte apoptosis is worthwhile. It may help us to more deeply clarify some aspects in thymic negative selection.

\section{ACKNOWLEDGMENTS}

The authors would like to thank Jia Ping TAO and Shi Liang MA for FACS sorting.

This work was funded by grants from Major State Bascic Research Development Program of China (No. G1999053904), National Natural Science Foundation of China (No. 30271200) and Young Scientist Cooperation Research Fund (No. 39928019).

Received, June 3, 2003

Revised, Jan 8, 2004

Accepted, Jan 12, 2004

\section{REFERENCES}

1 Anderson G, Moore NC, Owen JJT, Jenkinson EJ. Cellular interactions in thymocyte development. Annu Rev Immunol 1996; 14:73-99.

2 Sebzda E, Mariathasan S, Ohteki T, Jones R, Bachmann MF, Ohashi PS. Selection of the T cell repertoire. Annu Rev Immunol 1999; 17:829-74.

3 Brocker T, Riedinger M, Karjalainen K. Targeted expression of major histocompatibility complex (MHC) class II molecules demonstrates that dendritic cells can induce negative but not positive selection of thymocytes in vivo. J Exp Med 1997; 185 (3):541-50.

4 Anderson G, Partington KM, Jenkinson EJ. Differential effects 
of peptide diversity and stromal cell type in positive and negative selection in the thymus. J Immunol 1998; 161:6599-603.

5 Pircher H., K. Brduscha, U. Steinhoff, et al. Tolerance induction by clonal deletion of $\mathrm{CD} 4^{+} \mathrm{CD} 8^{+}$thymocytes in vitro does dot require dedicated antigen-presenting cells. Eur J Immunol 1993; 23:669-74.

6 Tanaka Y, Mamalaki C, Stockinger B, Kioussis D. In vitro negative selection of $\alpha \beta$ T cell receptor transgenic thymocytes by conditionally immortalized thymic cortical epithelial cell lines and dendritic cells. Eur J Immunol 1993; 23:2614-21.

7 Capone M, Romagnoli P, Beermann F, MacDonald HR, van Meerwijk JPM. Dissociation of thymic positive and negative selection in transgenic mice expressing major histocompatibility complex class I molecules exclusively on thymic cortical epithelial cells. Blood 2001; 97:1336-42.

8 Shu SZ, Chen WF, Liu ZG, Zhang PX, Wu JS. Establishment and identification of eight mouse thymic stromal cell lines. J. Beijing Medical University. (in Chinese) 1993; 25:237-41.

9 Pei LH, Liu ZG, Chen WF. Manipulation of mouse dendritic cell line clones and their characteristics. Chin J Immunol (in Chinese) 1999; 15:257-9.

10 Vremec D, Zorbas M, Scollay R, et al. The surface phenotype of dendritic cells purified from mouse thymus and spleen: Investigation of the CD8 expression by a subpopulation of dendritic cells. J Exp Med 1992; 176:47-58.

$11 \mathrm{Lu}$ LS, Chen WF. The functional development and the expression of Qa-2 antigen onthe medullary $\mathrm{CD}^{+} \mathrm{CD} 8^{-}$cells by the MTEC1 cells. Science in China (in Chinese) Ser B 1995; 25: 1161-6.

12 Dong HD, Pang XW, Chen WF. The different effects of two thymic stromal cells on programmed cell death of thymocytes cultured in vitro. Chin J Microbiol Immunol (in Chinese) 1996; 16:284-8.

13 Klein L, Kyewski B. Self-antigen presentation by thymic stromal cells: subtle division of labor. Curr Opin in Immunol 2000;
12:179-86.

14 Sebzda E, Wallace VA, Mayer J, eung RSMY, Mak TW, Ohashi PS. Positive and negative thymocyte selection induced by different concentrations of a single peptide. Science 1994; 263:1615-8.

15 Delaney JR, Sykulev Y, Eisen HN, Tonegawa S. Differences in the level of expression of class I major histocompatibility complex proteins on thymic epithelial and dendritic cells influence the decision of immature thymocytes between positive and negative selection. Proc Natl Acad Sci USA 1998; 95:5235-40.

16 Dong HD, Chen J, Chen WF. Membrane molecules in induction of apoptosis of thymocytes by mouse thymic dendritic cells which express Fas ligands. Science in China (Series C) 1998; 41: 189-94.

17 Liu ZG, Chen WF, Cao LX, Pang XW, Zhang PX, Yu Q. Cytokine production by MTSC4 cells, an established mouse thymic dendritic cell line. Acta Biologiae Experimentalis Sinica (in Chinese) 1994; 27:91-8.

18 Pang XW, Wu Q, Qian XP, Wu JS, Chen WF. Capability of antigen presentation by mouse thymic stromal cells. Chin $\mathrm{J}$ Microbiol Immunol (in Chinese) 2000; 20:28-31.

19 Surh CD, Sprent J. T-cell apoptosis detected in situ during positive and negative selection in the thymus. Nature 1994; 372: 100-3.

20 Guo J, Hawwari A, Li H, Sun Z, Mahanta SK. Regulation of the TCRalpha repertoire by the survival window of $\mathrm{CD}^{+} 8^{+}$ thymocytes. Nat Immunol 2002; 3:469-76.

21 Amsen,D. and A.M.Kruisbeek. Thymocyte selection: not by TCR alone. Immunological Reviews 1998; 165:209-29.

22 Lenschow DJ, Walunas TL, Bluestone JA. CD28/B7 system of T cell costimulation. Annu Rev Immunol 1996; 14:233-58.

23 Foy TM, Page DM, Waldschmidt TJ. et al. An essential role for gp39, the ligand for CD40, in thymic selection. J Exp Med 1995; 182:1377-88.

24 Kishimoto H, Surh CD, Sprent J. A role for Fas in negative selection of thymocytes in vivo. J Exp Med 1998; 187:1427-38. 